\title{
The backscattering ratio of comet 67P/Churyumov-Gerasimenko dust coma as seen by OSIRIS onboard Rosetta
}

I. Bertini, ${ }^{1 \star}$ F. La Forgia, ${ }^{1}$ M. Fulle ${ }^{\oplus},{ }^{2 \star}$ C. Tubiana ${ }^{\oplus},{ }^{3}$ C. Güttler ${ }^{\oplus},{ }^{3}$ F. Moreno,${ }^{4}$

J. Agarwal, ${ }^{3}$ O. Munoz, ${ }^{4}$ S. Mottola,${ }^{5}$ S. Ivanovsky, ${ }^{6,7}$ M. Pajola ${ }^{\odot},{ }^{8}$ A. Lucchetti, ${ }^{8}$ V. Petropoulou, ${ }^{9}$ M. Lazzarin, ${ }^{1}$ A. Rotundi, ${ }^{6,7}$ D. Bodewits, ${ }^{10}$ E. Frattin, ${ }^{1,8}$ I. Toth, ${ }^{11,12}$ N. Masoumzadeh, ${ }^{3}$ G. Kovacs, ${ }^{3}$ G. Rinaldi ${ }^{\oplus}, 6,7$ D. Guirado, ${ }^{4}$ H. Sierks, ${ }^{3}$ G. Naletto ${ }^{\odot}, 9,13,14$ P. Lamy, ${ }^{15}$ R. Rodrigo, ${ }^{16,17}$ D. Koschny, ${ }^{18}$ B. Davidsson, ${ }^{19}$ C. Barbieri, ${ }^{1}$ M. A. Barucci ${ }^{\oplus},{ }^{20}$ J.-L. Bertaux ${ }^{\oplus},{ }^{15}$ P. Cambianica, ${ }^{9}$ G. Cremonese, ${ }^{8}$ V. Da Deppo, ${ }^{14}$ S. Debei, ${ }^{21}$ M. De Cecco, ${ }^{22}$ J. Deller ${ }^{\oplus},{ }^{3}$ S. Ferrari, ${ }^{9}$ F. Ferri, ${ }^{9}$ S. Fornasier ${ }^{\oplus},{ }^{20}$ P. J. Gutierrez, ${ }^{4}$ P. H. Hasselmann, ${ }^{20}$ W.-H. Ip,${ }^{23,24}$ H. U. Keller, ${ }^{5,25}$ L. M. Lara, ${ }^{4}$ J. J. Lopez Moreno, ${ }^{4}$ F. Marzari, ${ }^{13}$ M. Massironi,,${ }^{9,26}$ L. Penasa ${ }^{\oplus 9}$ and $\mathrm{X} . \mathrm{Shi}^{3}$

Affiliations are listed at the end of the paper

Accepted 2018 October 18. Received 2018 October 18; in original form 2018 June 8

\begin{abstract}
Remote sensing observations of dust particles ejected from comets provide important hints on the intimate nature of the materials composing these primitive objects. The measurement of dust coma backscattering ratio, $B S R$, defined as the ratio of the reflectance at phase angle $0^{\circ}$ and $30^{\circ}$, helps tuning theoretical models aimed at solving the inverse scattering problem deriving information on the nature of the ejected particles. The Rosetta/OSIRIS camera sampled the coma phase function of comet 67P, with four series acquired at low phase angles from 2015 January to 2016 May. We also added previously published data to our analysis to increase the temporal resolution of our findings. We measured a $B S R$ in the range $\sim$ [1.7-3.6], broader than the range found in literature from ground-based observations of other comets. We found that during the post-perihelion phase, the $B S R$ is systematically larger than the classical cometary dust values only for nucleocentric distances smaller than $\sim 100 \mathrm{~km}$. We explain this trend in terms of a cloud of chunks orbiting the nucleus at distances $<100 \mathrm{~km}$ ejected during perihelion and slowly collapsing on the nucleus over a few months because of the coma gas drag. This also implies that the threshold particle size for the dust phase function to become similar to the nucleus phase function is between $2.5 \mathrm{~mm}$ and $0.1 \mathrm{~m}$, taking into account previous Rosetta findings.
\end{abstract}

Key words: scattering-methods: data analysis - techniques: image processing - techniques: photometric-comets: individual: 67P/Churyumov-Gerasimenko.

\section{INTRODUCTION}

Refractories constitute the largest massive component released by comet 67P/Churyumov-Gerasimenko, hereafter 67P, in its path in the Solar system (Fulle et al. 2016a, and references therein). Remote sensing observations of the dust particles assume therefore a pivotal importance in studying the intimate nature of the materials composing the primitive comet.

The measurement of the dust coma backscattering ratio at low phase angles is pivotal in tuning theoretical models aimed at solving the inverse scattering problem deriving information on the nature of the ejected particles, providing also consequent hints on the dust albedo.

Four OSIRIS multiwavelength series were acquired in 2015 January, 2016 February, 2016 April, and 2016 May to study the 
Table 1. Filters used in the phase function analysis.

\begin{tabular}{lccr}
\hline Camera & Filter name & $\begin{array}{c}\text { Peak transmission } \\
\text { wavelength }(\mathrm{nm})\end{array}$ & $\begin{array}{c}\text { Bandwidth } \\
(\mathrm{nm})\end{array}$ \\
\hline WAC & UV375 F13 & 375.6 & 9.8 \\
WAC & Green F21 & 537.2 & 63.2 \\
WAC & Vis610 F18 & 612.6 & 9.8 \\
WAC & Red F12 & 629.8 & 156.8 \\
NAC & Blue F24 & 480.7 & 74.9 \\
NAC & Orange F22 & 649.2 & 84.5 \\
NAC & Red F28 & 743.7 & 64.1 \\
\hline
\end{tabular}

backscattering intensity of solar light scattered by the coma dust. We added to our analysis also the image series described in Bertini et al. (2017), although obtained starting from larger observational phase angles, to enlarge the temporal coverage of our findings.

The results of our analysis in terms of the dust backscattering ratio measurement versus time and nucleocentric distance are presented in the following sections and interpreted in terms of hints on the nature of the observed particles. We also compared our findings to literature results, regarding both cometary comae and 67P nucleus.

\section{OBSERVATIONS}

Four Rosetta/OSIRIS (Keller et al. 2007) image series were acquired between 2015 January 9 (heliocentric distance, $r_{\mathrm{h}}=2.58$ au inbound) and 2016 May $9\left(r_{\mathrm{h}}=2.97\right.$ au outbound), with a perihelion date of 2015 August 13, in order to measure the backscattering ratio of the dust coma with multiwavelength observations. We obtained several Narrow Angle Camera (NAC) and Wide Angle Camera (WAC) observations using narrow-band and broad-band filters from 375.6 to $743.7 \mathrm{~nm}$ (Table 1). The WAC narrow-band filters are sampling the continuum of the coma spectrum where no gaseous emission lines are expected. We assumed that the gaseous emissions in the broad-band filters have a minor contribution to the total integrated signal, as in Bertini et al. (2017). Our measurements refer therefore to the solar light scattered by dust particles in the coma. The log of the observations is shown in Table 2.

All series were acquired in a similar way, maintaining, within one single series, the nucleus elongation $E_{\mathrm{n}}$, defined as the angle between the Sun and the nucleus as seen from the spacecraft (according to the classical astronomical notation), to a fixed value (usually around $90^{\circ}$ ). The nucleus scattering plane is the plane containing the nucleus, the Sun, and the spacecraft Rosetta (Panel (a) in Fig. 1). From the infinite number of coma scattering planes we picked the plane perpendicular to the plane containing the Sun, the comet nucleus and Rosetta, as well as containing the Sun direction. The angle for observation $i$, between the telescope boresight and the vector from the dust particles to the Sun is the phase angle $\alpha_{i}$ for a particular observation (Panel (b) in Fig. 1).

This observing strategy allowed measuring in the four aforementioned series the coma reflectivity sampling a range in phase angle up to $\sim 60^{\circ}$ in the worst case and up to $\sim 150^{\circ}$ in the best case in a very short amount of time (lower than $2 \mathrm{~h}$ ), thus minimizing the assumption of observing dust with the same intimate nature at different pointing stations. Series dedicated to the measurement of the coma phase function were obtained throughout the entire Rosetta mission, as described in Bertini et al. (2017). Nevertheless, pointing at $\alpha<15^{\circ}$ (solar elongation $>165^{\circ}$ ) was possible only when Rosetta was beyond 2.21 au from the Sun (before 2015 February and after 2016 February) due to thermal constraints of the spacecraft. This resulted in scanning the backscattering ratio of the coma at the initial and final months of the mission.

The log of the observations at low phase angle is given in Table 2, while we refer the reader to Bertini et al. (2017) for an analogous table of the complementary series taken starting from larger phase angles.

\section{DATA REDUCTION}

All data were calibrated photometrically in reflectance, $R=\pi I /\left(F_{\odot} / r_{h}^{2}\right)$, where $I$ is the measured spectral irradiance and $F_{\odot}$ is the solar flux at the observation heliocentric distance $r_{\mathrm{h}}$, using the instrumental pipeline described in Tubiana et al. (2015).

The data reduction procedure is very similar to the one described in Bertini et al. (2017). We used the NASA ancillary NAIF-SPICE system kernels (Acton 1996; Acton et al. 2018), containing all the necessary geometric and temporal parameters specific for Rosetta, to obtain the relative position of the nucleus, the Sun, the spacecraft, the boresight of the camera, and the relative angles at any specific moment. Additionally, all the optical parameters of the camera were used, such as field of view, resolution and pixel sizes, and combined with the observing geometry to generate a phase angle map corresponding to each image, allowing us to associate a phase angle value to each pixel of the image. Examples of phase angle maps are shown for a WAC and an NAC image in Figs 2(b) and (e) with overplotted contour lines. Then we divided the image in anular sections following the profile of the phase maps and having a fixed width of $1^{\circ}$ (see Fig. 2). In this way we were able to sample the entire images in phase angle.

Finally, we retrieved the reflectance value versus the phase angle of observation for each anular section using the IDL mmm.pro

Table 2. OSIRIS image series dedicated to the coma backscattering ratio measurement. MTP, STP, $r_{\mathrm{h}}, r_{\mathrm{c}}, t_{\mathrm{start}}$, and $t_{\text {exp }}$ stand for Medium Term Planning (duration 1 month), Short Term Planning (duration 1 week) from the Rosetta hibernation exit on 2014 January 20, heliocentric distance, nucleocentric distance, time of exposure start, and exposure duration, respectively.

\begin{tabular}{lcccccc}
\hline Series (MTP/STP) & Date & Filters & $r_{\mathrm{h}}(\mathrm{au})$ & $r_{\mathrm{c}}(\mathrm{km})$ & $t_{\text {start }}(\mathrm{UT})$ & $t_{\exp }(\mathrm{s})$ \\
\hline $011 / 038$ & $09 / 01 / 2015$ & WAC F18, F21 & 2.58 & 27 & $14: 52: 11$ & $0.51,0.09$ \\
$026 / 096$ & $18 / 02 / 2016$ & WAC F21 & 2.39 & 35 & $12: 26: 27$ & 0.45 \\
& & NAC F22, F24, F28 & & & & $14,146,80$ \\
$027 / 102$ & WAC F21 & 2.73 & 320 & $03: 36: 46$ & 4.5 \\
& $04 / 04 / 2016$ & NAC F22, F24 & & & & 70,146 \\
$029 / 107$ & $09 / 05 / 2016$ & WAC F12, F21 & 2.97 & 17 & $18: 02: 14$ & $1.5,4.5$ \\
& & NAC F22, F24 & & & & 70,146 \\
\hline
\end{tabular}



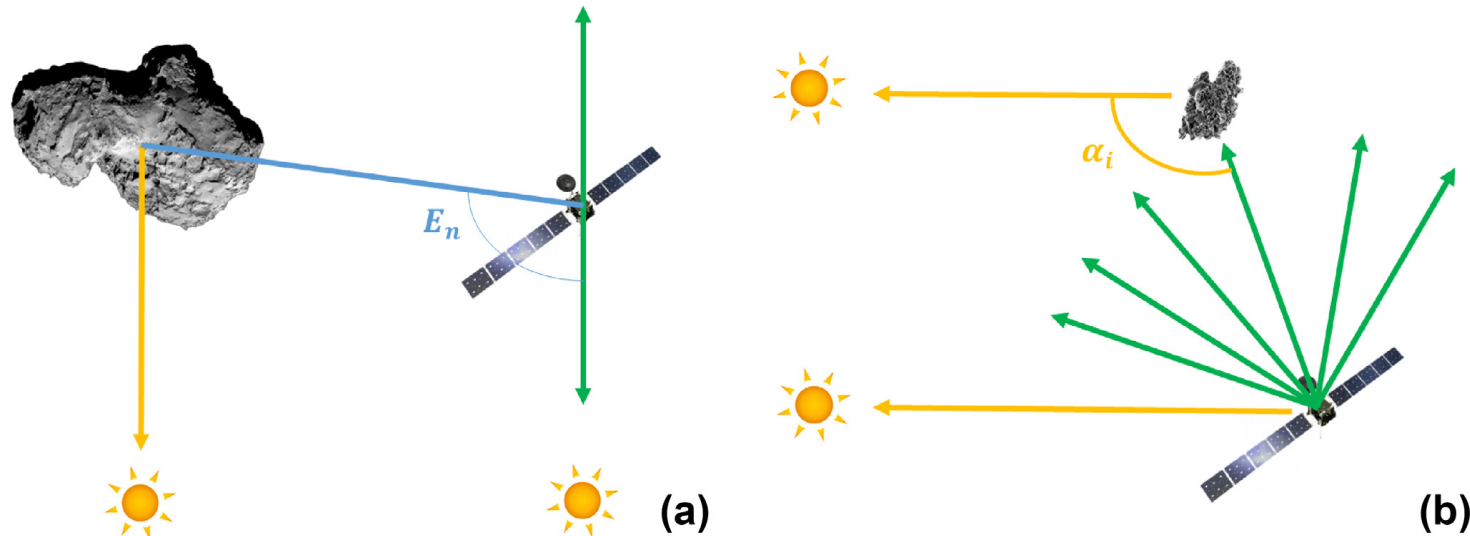

Figure 1. Observational geometry of the OSIRIS phase function series. Panel (a) depicts the geometry in the comet-spacecraft-Sun plane. $E_{\mathrm{n}}$ is the nucleus elongation angle. Panel (b) shows the configuration of a plane perpendicular to the aforementioned plane and also containing the direction towards the Sun. We chose this as our scattering plane. It contains the Sun, the spacecraft, and the dust. An example of a dust particle is shown at the top of Panel (b). The different green arrows indicate the telescope boresight pointing directions. We indicate the phase angle $\alpha_{i}$ for the $i$-th pointing direction as an example.
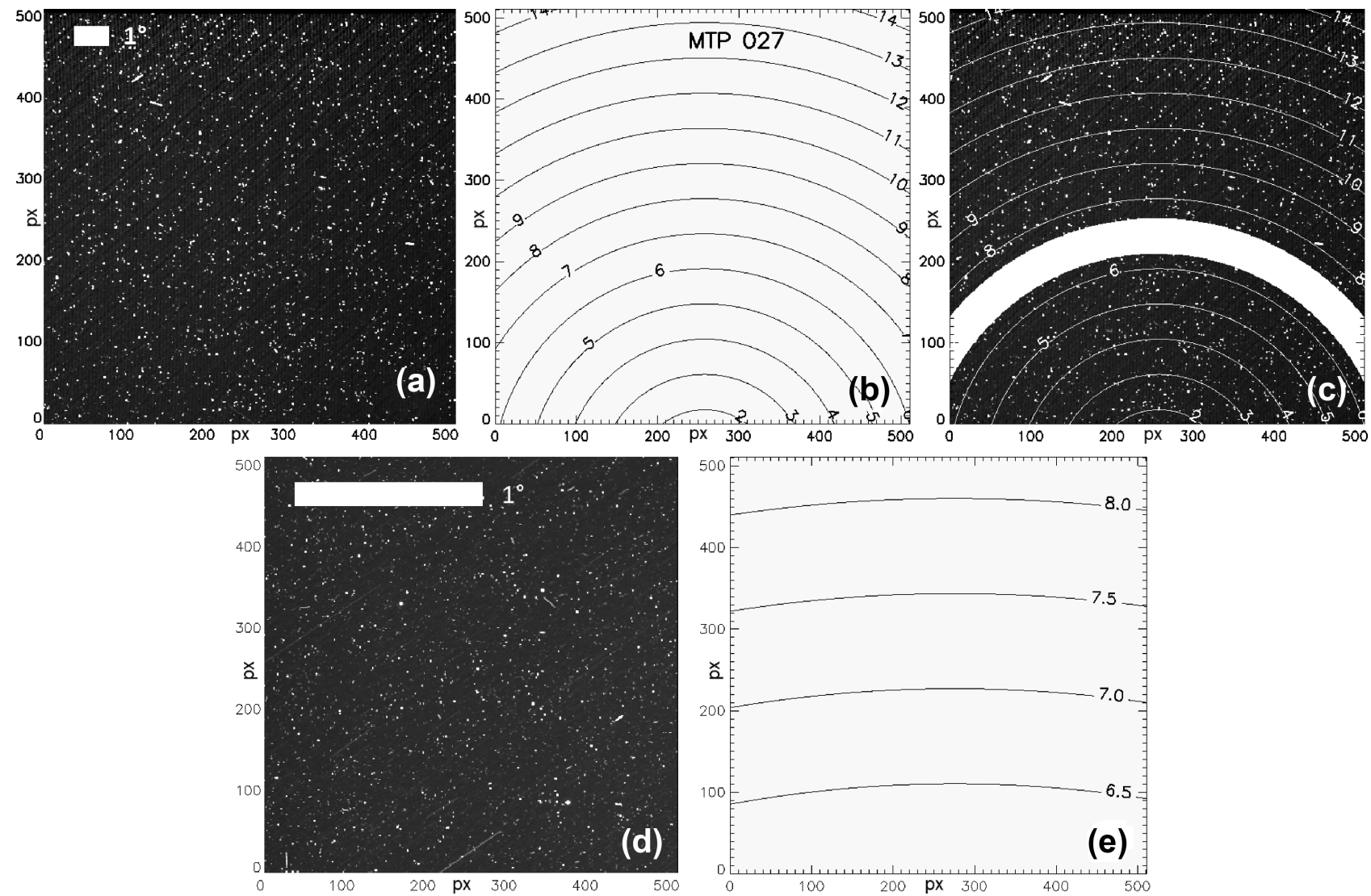

Figure 2. OSIRIS MTP027 images at low phase angle (pointing at $\alpha=7.1^{\circ}$ ) with correspondent phase maps. Sample WAC image (a), correspondent phase angle map (b), and sample anular section where the background signal was measured (c). Sample NAC image (d) and correspondent phase angle map (e). Dots and strikes are cosmic ray hits and dust particles tracks during the exposure.

procedure to calculate the signal level of the optical background, getting rid of the contaminating stars, cosmic ray hits, and grain tracks with large positive values. The reflectance value at a phase angle of value $X$ was measured within the anular section comprised between $X-0.5^{\circ}$ and $X+0.5^{\circ}$.

\section{THE COMA BACKSCATTERING RATIO}

In the analysis of the coma phase function in the backscattering region we considered the measured reflectance in the phase angle range $\left[0^{\circ}-90^{\circ}\right]$, avoiding the problem of the forward scattering straylight contamination described in Bertini et al. (2017). 

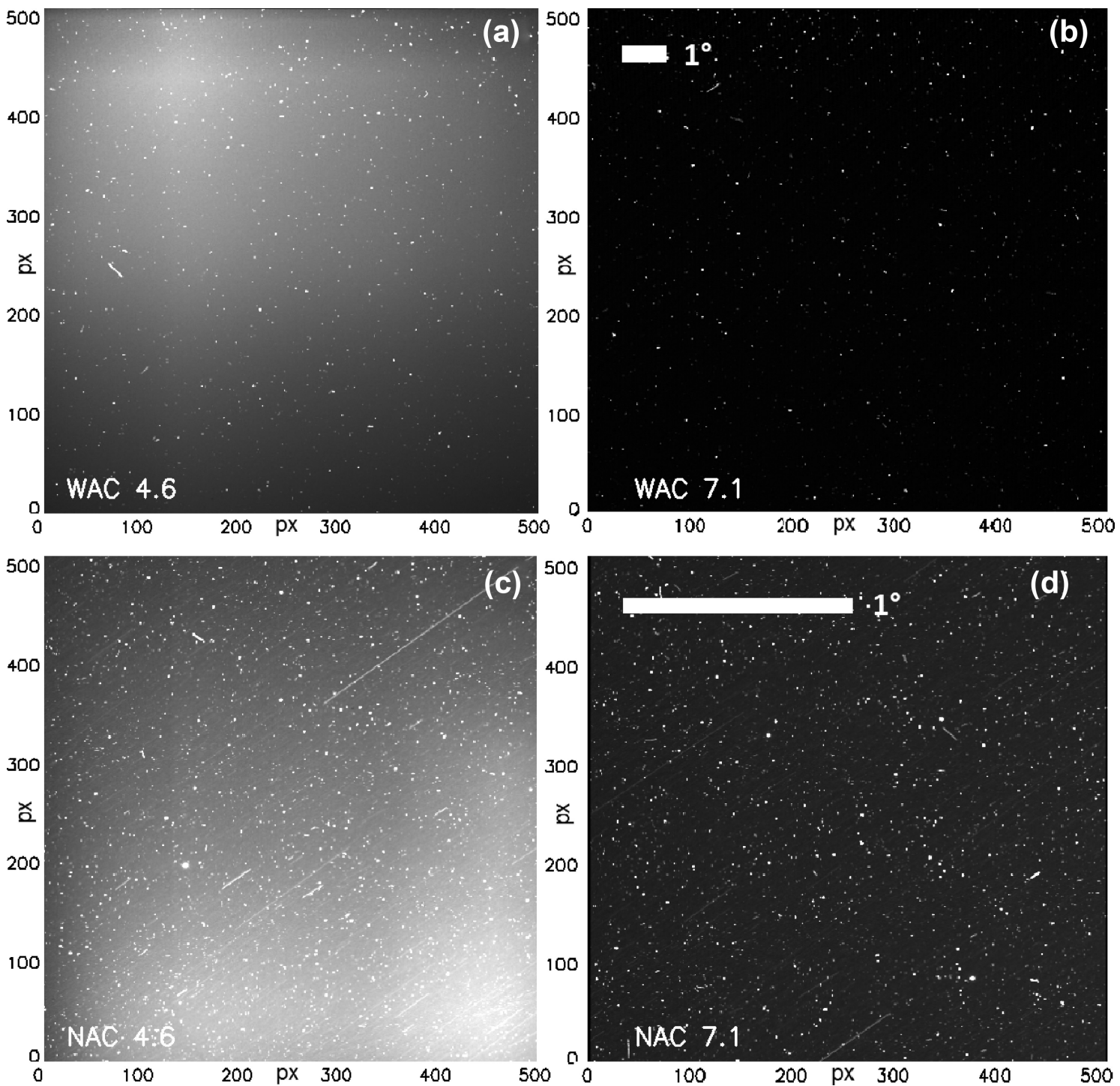

Figure 3. Straylight contribution in OSIRIS images at small phase angles. In panels (a) and (b), WAC images during MTP027 and with a pointing (centre of the image) corresponding to $\alpha=4.6^{\circ}$ and $\alpha=7.1^{\circ}$ are shown, respectively. The same effect is shown in MTP027 NAC images in panels (c) and (d)

The increase of scattered intensity in the backscattering direction, or back scattering ratio $B S R$, can be measured as the ratio of the phase function at $\alpha=0^{\circ}$ and $\alpha=30^{\circ}$ (Bertini, Thomas \& Barbieri 2007, and references therein), i.e. in our case as $B S R=R\left(0^{\circ}\right) / R\left(30^{\circ}\right)$.

Unfortunately, we discovered an additional straylight contribution due to an unknown source affecting images acquired at pointings lower than $7^{\circ}$ of phase angle. Although the physical cause is still under investigation, a possible source may be the brightness of the coma itself at opposition producing ghost and glow effects through the optical system (Dohlen et al. 2010). The aforementioned contamination was found both with visual inspections (low phase angle data show sections of the image with clear straylight patterns) and plotting the reflectance obtained in small square sections against the phase angle. The contaminated sections produce a dichotomy of the signal at same phase angle with respect to clean sections. This dichotomy disappears for pointings greater than or equal to $7^{\circ}$. In Fig. 3 we plot MTP027 WAC and NAC images comparing with the same visualization stretch the pointings at $\alpha=4.6^{\circ}$, where straylight patterns are clearly visible, with the subsequent pointings at $\alpha=7.1^{\circ}$, where the straylight is not present anymore.
Images starting with pointings of $7^{\circ}$ on are therefore free from contamination sources and provide, applying the correspondent phase map, a clean source of the signal down to phase angles $\sim 2^{\circ}$ in the best WAC case. In the best NAC case, because of the design of the signal extraction procedure, the clean signal with lowest phase angle is placed at $7^{\circ}$ (Fig. 2).

We then extrapolated our findings at $0^{\circ}$ of phase angle using a third-order polynomial fit of our measurements between the minimum available phase angle data and a maximum of $\sim 60^{\circ}$, this last value being determined for consistency with the smallest extension in phase angle observed in some considered series. The polynomial is characterized by the equation:

$R(\alpha)=\sum_{k=0}^{n} A_{k} \alpha^{k}$.

A polynomial was chosen because it provides a fit with lower residuals with respect to exponential ones. The third degree was considered giving a good standard deviation uncertainty minimizing the free parameters to fit. Although cometary comae phase 

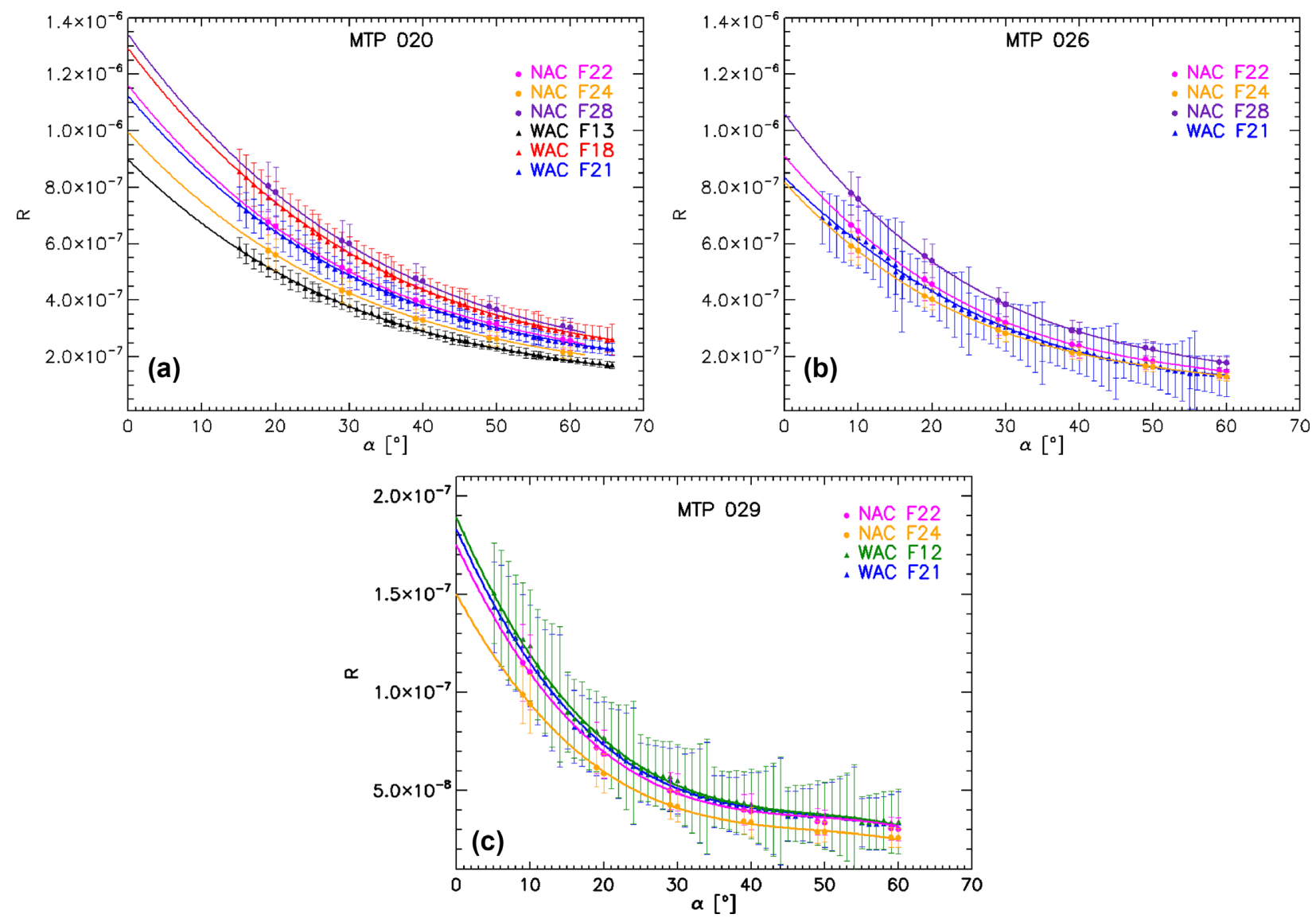

Figure 4. Third-order best polynomial fit curves for multiwavelength OSIRIS MTP020 (a), MTP026 (b), and MTP029 (c) reflectivity data.

curves may not always behave like simple polynomials, we used the polynomial fit to interpolate our measurements as a best way to provide the desired extrapolation at $0^{\circ}$ of phase angle which was not possible to have directly from the measurements. The polynomial, or exponential, fit of the measured phase function is an approach already used in literature (e.g. Fulle et al. 2018; Gerig et al. 2018). Sample measurements together with the polynomial fit curves are portrayed in Fig. 4 for all filters used in MTP020, MTP026, and MTP029 image series. The coefficients of the third-order polynomial fit for the images in WAC F18 and NAC F22 filters throughout our observations are listed in Table 3.

We then measured the BSR in different filters, varying the heliocentric and nucleocentric distance pre- and post-perihelion, using the correspondent values given by equation (1). Our results are shown in Table 4. The error on the BSR measurement was calculated propagating the error provided by the polynomial fit itself.

We first note that the measurements of the backscattering ratio taken at different wavelengths and with different filters are clustered around similar values within one single series. This implies: (1) absence of phase reddening in the $B S R$ range as already showed in Bertini et al. (2017) up to $\alpha=90^{\circ}$, (2) constant dust colours for $\alpha$ $\leq 30^{\circ}$, and (3) likely small gas contamination if any because this contamination would have affected differentially the measurements taken at different wavelengths.

We then interpreted the results in terms of days from perihelion passage (Fig. 5) and cometocentric distance.

A scientific interpretation of our findings cannot be separated from an initial comparison with the results in literature coming from the coma of other comets, the dust in the interplanetary environment, and on the nucleus of 67P itself, although these results were obtained with a wide range of filters, cometocentric, and heliocentric distances. Below we report the results of such a literature search. We also underline that the following values are derived from fits performed following different methodologies, without the claim of referring to uniform results.

Meech \& Jewitt (1987) found a $B S R=2.51,1.72$, and 2.63 for the Jupiter family comet 47P/Ashbrook-Jackson, the Halley-type comet 38P/Stephan-Oterma, and the hyperbolic comet C/1980 E1 (Bowell), respectively. Millis, Ahearn \& Thompson (1982) measured $B S R \sim 1.95$ for 38P/Stephan-Oterma. The Jupiter family comet 22P/Kopff has $B S R=2.19$ (Moreno et al. 2012). The interplanetary dust has a $B S R=1.61$ (Ishiguro et al. 2013). Overall values between $\sim 1.7$ and $\sim 2.6$ define the backscattering behaviour of cometary comae dust particles, according to previous literature studies, slightly larger than the characteristic value of interplanetary dust. We consider $B S R=2.6$ as an upper limit threshold defining cometary dust as seen from ground-based observations so far. Interestingly, Frattin \& et (2018) found a BSR below 2.27 in laboratory scattering experiments involving micron-sized cometary dust analogues (fine-grained primitive meteorites). This would imply that small values of $B S R$ characterize small micron-sized particles.

The nucleus is instead characterized by larger values of the backscattering ratio. Güttler et al. (2017), extending data from Fornasier et al. (2015), found $B S R=3.86$ for the unresolved $67 \mathrm{P}$ nucleus in the NAC F22 filter, while Hasselmann et al. (2017) measured a $B S R=3.59$ in the WAC F18 filter considering all terrains by 
Table 3. Third-order polynomial fit coefficients for the WAC F18 and NAC F22 filters.

\begin{tabular}{lccccc}
\hline Series (MTP/STP) & Filters & $A_{0}$ & $A_{1}$ & $A_{2}$ & $A_{3}$ \\
\hline $011 / 038$ & WAC F18 & $6.06 \mathrm{E}-07$ & $-2.02 \mathrm{E}-08$ & $3.94 \mathrm{E}-10$ & $-2.66 \mathrm{E}-12$ \\
$014 / 049$ & WAC F18 & $2.94 \mathrm{E}-06$ & $-9.75 \mathrm{E}-08$ & $1.33 \mathrm{E}-09$ & $-6.27 \mathrm{E}-12$ \\
$018 / 063$ & NAC F22 & $6.46 \mathrm{E}-07$ & $-1.81 \mathrm{E}-08$ & $2.84 \mathrm{E}-10$ & $-1.73 \mathrm{E}-12$ \\
& WAC F18 & $7.51 \mathrm{E}-07$ & $-1.95 \mathrm{E}-08$ & $2.67 \mathrm{E}-10$ & $-1.35 \mathrm{E}-12$ \\
$019 / 070$ & WAC F18 & $6.44 \mathrm{E}-07$ & $-1.78 \mathrm{E}-08$ & $2.49 \mathrm{E}-10$ & $-1.40 \mathrm{E}-12$ \\
$020 / 071$ & NAC F22 & $1.16 \mathrm{E}-06$ & $-3.27 \mathrm{E}-08$ & $4.22 \mathrm{E}-10$ & $-2.14 \mathrm{E}-12$ \\
$021 / 075$ & WAC F18 & $1.29 \mathrm{E}-06$ & $-3.44 \mathrm{E}-08$ & $3.94 \mathrm{E}-10$ & $-1.66 \mathrm{E}-12$ \\
$022 / 081$ & NAC F22 & $4.91 \mathrm{E}-07$ & $-1.71 \mathrm{E}-08$ & $2.73 \mathrm{E}-10$ & $-1.67 \mathrm{E}-12$ \\
$023 / 083$ & NAC F22 & $4.78 \mathrm{E}-07$ & $-1.52 \mathrm{E}-08$ & $2.36 \mathrm{E}-10$ & $-1.34 \mathrm{E}-12$ \\
$023 / 086$ & NAC F22 & $1.74 \mathrm{E}-06$ & $-4.39 \mathrm{E}-08$ & $7.06 \mathrm{E}-10$ & $-5.13 \mathrm{E}-12$ \\
$024 / 090$ & NAC F22 & $1.06 \mathrm{E}-06$ & $-3.96 \mathrm{E}-08$ & $6.65 \mathrm{E}-10$ & $-4.24 \mathrm{E}-12$ \\
$025 / 092$ & NAC F22 & $5.02 \mathrm{E}-07$ & $-1.89 \mathrm{E}-08$ & $3.07 \mathrm{E}-10$ & $-1.86 \mathrm{E}-12$ \\
$026 / 096$ & NAC F22 & $2.77 \mathrm{E}-07$ & $-9.84 \mathrm{E}-09$ & $1.76 \mathrm{E}-10$ & $-1.1 \mathrm{E}-12$ \\
$027 / 102$ & NAC F22 & $9.10 \mathrm{E}-07$ & $-3.06 \mathrm{E}-08$ & $4.37 \mathrm{E}-10$ & $-2.31 \mathrm{E}-12$ \\
$029 / 107$ & NAC F22 & $6.55 \mathrm{E}-08$ & $-1.12 \mathrm{E}-09$ & $-3.02 \mathrm{E}-12$ & $1.22 \mathrm{E}-13$ \\
\hline
\end{tabular}

applying a third-order polynomial fit to the published data. Finally, Masoumzadeh et al. (2017) obtained an average value of $B S R=$ 4.16, considering data of a local region located at the Imhotep-Ash boundary in the NAC F24, F22, and F28 filters.

Our results do not show a clear trend versus time (Fig. 5) and provide a measurement of the $B S R$ in $67 \mathrm{P}$ coma in the range $\sim[1.7-$ 3.6], a broader interval with respect to previous published results on cometary comae at larger nucleocentric distances.

\section{DISCUSSION}

Fulle et al. (2018) have shown that the dust phase function described by Bertini et al. (2017) is valid up to dust sizes of $2.5 \mathrm{~mm}$. This has been confirmed by recent light scattering calculations of absorbing (refractive index of $m=1.6+0.1 i$ ) and large respect to visible wavelengths (from $10 \mu \mathrm{m}$ to $\mathrm{mm}$-sized) particles, being distributed in a wide variety of aspect ratios (spheroidally shaped particles having axial ratios ranging from 0.25 to 4 ). These calculations have been performed with T-matrix, Multiple Sphere T-matrix, and raytracing methods (Moreno et al. 2018). The authors show that the Bertini et al. (2017) phase function shape is maintained from 10 $\mu \mathrm{m}$ up to $\mathrm{mm}$-sized particles in the $20^{\circ}-110^{\circ}$ phase angle domain [which is the range used in Fulle et al. (2018)]. The 67P coma dust size distribution shows a strong time evolution below sizes of about $1 \mathrm{~mm}$ (Fulle et al. 2016b). However, the dominant cross-section is always $<2.5 \mathrm{~mm}$ during the entire mission (Rotundi et al. 2015; Fulle et al. 2016b), so that any BSR time evolution cannot be due to the time evolution of 67P dust size distribution observed by GIADA (Della Corte et al. 2014) and OSIRIS at a nucleus distance larger than $100 \mathrm{~km}$ (Fulle et al. 2016a).

The nucleus $B S R$ places a clear upper limit on the dust $B S R$. After perihelion, we found a clear trend comparing the dust $B S R$ and the Rosetta distance $r_{\mathrm{c}}$ from the nucleus centre (Fig. 6): the dust $B S R$ is larger than the classical values for cometary dust only for nucleocentric distances smaller than $\sim 100 \mathrm{~km}$, with the largest $B S R$ value, close to the nucleus one, corresponding to the smallest nucleocentric distance $(17 \mathrm{~km})$. There is the caveat of MTP21 taken at large nucleocentric distance $(1200 \mathrm{~km})$ and exhibiting a $B S R$ larger than our defined threshold. A possible explanation may come from the fact that due to the solar radiation pressure, the dust space density is the more isotropic, the farther the dust is from the nucleus. The BSR (larger than average) observed at $1200 \mathrm{~km}$, when Rosetta was observing with a nucleus elongation angle of about $130^{\circ}$, may be also due to the optical depth crossing a spherically symmetric coma. Models for a backscattering pointing compute a $B S R$ increase of 10 per cent at most.

We explain the systematic post-perihelion $B S R$ versus $r_{\mathrm{c}}$ trend in terms of a cloud of chunks orbiting the $67 \mathrm{P}$ nucleus at distances $<100 \mathrm{~km}$. We note that also the chuncks observed around the Jupiter-family comet 103P/Hartley 2 may be mostly in bound orbits (Kelley et al. 2013), that we will show here to be necessarily collapsing on the nucleus due to coma gas drag. During perihelion, $67 \mathrm{P}$ ejected several thousands of $\mathrm{kg} \mathrm{s}^{-1}$ of dm-sized chunks (Fulle et al. 2016b; Ott et al. 2017), probably scattering light similarly to the 67P nucleus (Fulle et al. 2018). The chunks ejected sunward were decelerating below their escape speed due to outgassing (Agarwal et al. 2016) which may be consistent with a negligible global water distributed source, thus entering into bound orbits of e.g. semimajor axis $a=50 \mathrm{~km}$ and period of about 1 month. The chunk orbit is perturbed by the gas drag in the coma. Gauss orbital perturbations are

$$
\begin{aligned}
& \frac{\mathrm{d} a}{\mathrm{~d} \theta}=\frac{2 a^{2}}{\sqrt{\left(1-e^{2}\right) a \mathrm{GM}}}\left[e A_{r} \sin \theta+(1+e \cos \theta) A_{\theta}\right] \\
& \frac{\mathrm{d} e}{\mathrm{~d} \theta}=\sqrt{\frac{\left(1-e^{2}\right) a}{\mathrm{GM}}}\left[A_{r} \sin \theta+\frac{2 \cos \theta+e+e \cos ^{2} \theta}{1+e \cos \theta} A_{\theta}\right],
\end{aligned}
$$

where $e$ is the orbital eccentricity, $\theta$ is the orbital true anomaly, and $\mathrm{GM}=667 \mathrm{~m}^{3} \mathrm{~s}^{-2}$ for 67P. $A_{r}$ and $A_{\theta}$ are the radial and tangential perturbations to the chunk motion. When we integrate equations (2) and (3) over a whole orbit, all the terms depending on $A_{r}$ become 
Table 4. Dust coma backscattering ratio final results. $r_{\mathrm{h}}, r_{\mathrm{c}}, \alpha$, and $E_{\mathrm{n}}$ stand for heliocentric distance, cometocentric distance, phase angle range of the dust coma considered for the polynomial fit, and nucleus elongation range during the time span of the reported observations, respectively. The double continuous line in the table separates the observations taken before and after perihelion.

\begin{tabular}{|c|c|c|c|c|c|c|c|}
\hline $\begin{array}{l}\text { Series } \\
\text { (MTP/STP) }\end{array}$ & Date & Filters & $r_{\mathrm{h}}(\mathrm{au})$ & $r_{\mathrm{c}}(\mathrm{km})$ & $\alpha\left(^{\circ}\right)$ & $E_{\mathrm{n}}\left({ }^{\circ}\right)$ & $B S R$ \\
\hline $011 / 038$ & 09/01/2015 & $\begin{array}{l}\text { WAC F18 } \\
\text { WAC F21 }\end{array}$ & 2.58 & 27 & $\begin{array}{l}5-56 \\
6-60\end{array}$ & 90 & $\begin{array}{l}2.14 \pm 0.05 \\
1.92 \pm 0.06\end{array}$ \\
\hline $014 / 049$ & $29 / 03 / 2015$ & $\begin{array}{l}\text { WAC F13 } \\
\text { WAC F18 } \\
\text { WAC F21 }\end{array}$ & 1.98 & 60 & $\begin{array}{l}10-56 \\
10-56 \\
10-56\end{array}$ & 100 & $\begin{array}{l}3.04 \pm 0.03 \\
2.83 \pm 0.03 \\
2.99 \pm 0.04\end{array}$ \\
\hline $018 / 063$ & $07 / 07 / 2015$ & $\begin{array}{l}\text { NAC F22 } \\
\text { NAC F24 } \\
\text { WAC F13 } \\
\text { WAC F18 } \\
\text { WAC F21 }\end{array}$ & 1.32 & 153 & $\begin{array}{l}19-60 \\
19-60 \\
15-66 \\
15-66 \\
15-66\end{array}$ & 90 & $\begin{array}{l}2.07 \pm 0.03 \\
2.12 \pm 0.02 \\
2.03 \pm 0.02 \\
2.04 \pm 0.02 \\
2.02 \pm 0.02\end{array}$ \\
\hline 019/070 & $20 / 08 / 2015$ & $\begin{array}{l}\text { WAC F13 } \\
\text { WAC F18 } \\
\text { WAC F21 }\end{array}$ & 1.24 & 320 & $\begin{array}{l}15-66 \\
15-66 \\
15-66\end{array}$ & 90 & $\begin{array}{l}2.19 \pm 0.01 \\
2.16 \pm 0.02 \\
2.14 \pm 0.02\end{array}$ \\
\hline 020/071 & $28 / 08 / 2015$ & $\begin{array}{l}\text { NAC F22 } \\
\text { NAC F24 } \\
\text { NAC F28 } \\
\text { WAC F13 } \\
\text { WAC F18 } \\
\text { WAC F21 }\end{array}$ & 1.25 & 420 & $\begin{array}{l}19-60 \\
19-60 \\
19-60 \\
15-66 \\
15-66 \\
15-66\end{array}$ & 108 & $\begin{array}{l}2.31 \pm 0.01 \\
2.34 \pm 0.01 \\
2.24 \pm 0.01 \\
2.36 \pm 0.01 \\
2.28 \pm 0.02 \\
2.30 \pm 0.02\end{array}$ \\
\hline $021 / 075$ & $28 / 09 / 2015$ & $\begin{array}{l}\text { NAC F22 } \\
\text { NAC F24 } \\
\text { NAC F28 }\end{array}$ & 1.37 & 1200 & $\begin{array}{l}19-50 \\
19-50 \\
19-50\end{array}$ & 130 & $\begin{array}{l}2.76 \pm 0.01 \\
2.82 \pm 0.01 \\
2.76 \pm 0.01\end{array}$ \\
\hline $022 / 081$ & $06 / 11 / 2015$ & $\begin{array}{l}\text { NAC F22 } \\
\text { NAC F24 } \\
\text { NAC F28 }\end{array}$ & 1.60 & 240 & $\begin{array}{l}19-60 \\
19-60 \\
19-60\end{array}$ & 117 & $\begin{array}{l}2.40 \pm 0.02 \\
2.46 \pm 0.01 \\
2.18 \pm 0.02\end{array}$ \\
\hline $023 / 083$ & $19 / 11 / 2015$ & $\begin{array}{l}\text { NAC F22 } \\
\text { NAC F24 } \\
\text { NAC F28 }\end{array}$ & 1.69 & 125 & $\begin{array}{l}19-60 \\
19-60 \\
19-60\end{array}$ & 105 & $\begin{array}{l}1.89 \pm 0.01 \\
1.79 \pm 0.01 \\
1.73 \pm 0.01\end{array}$ \\
\hline 023/086 & $14 / 12 / 2015$ & $\begin{array}{l}\text { NAC F22 } \\
\text { NAC F24 } \\
\text { NAC F28 }\end{array}$ & 1.88 & 100 & $\begin{array}{l}19-60 \\
19-60 \\
19-60\end{array}$ & 91 & $\begin{array}{l}2.97 \pm 0.02 \\
2.71 \pm 0.01 \\
2.64 \pm 0.01\end{array}$ \\
\hline 024/090 & $07 / 01 / 2016$ & $\begin{array}{l}\text { NAC F22 } \\
\text { NAC F24 } \\
\text { NAC F28 }\end{array}$ & 2.07 & 75 & $\begin{array}{l}19-60 \\
19-60 \\
19-60\end{array}$ & 90 & $\begin{array}{l}3.12 \pm 0.02 \\
3.05 \pm 0.02 \\
2.99 \pm 0.02\end{array}$ \\
\hline $025 / 092$ & $21 / 01 / 2016$ & $\begin{array}{l}\text { NAC F22 } \\
\text { NAC F24 } \\
\text { NAC F28 }\end{array}$ & 2.18 & 80 & $\begin{array}{l}19-60 \\
19-60 \\
19-60\end{array}$ & 118 & $\begin{array}{l}2.49 \pm 0.01 \\
2.64 \pm 0.02 \\
2.54 \pm 0.02\end{array}$ \\
\hline 026/096 & $18 / 02 / 2016$ & $\begin{array}{l}\text { NAC F22 } \\
\text { NAC F24 } \\
\text { NAC F28 } \\
\text { WAC F21 }\end{array}$ & 2.39 & 35 & $\begin{array}{l}9-60 \\
9-60 \\
9-60 \\
5-60\end{array}$ & 116 & $\begin{array}{l}2.81 \pm 0.02 \\
2.87 \pm 0.02 \\
2.73 \pm 0.02 \\
2.73 \pm 0.08\end{array}$ \\
\hline $027 / 102$ & $04 / 04 / 2016$ & $\begin{array}{l}\text { NAC F22 } \\
\text { NAC F24 } \\
\text { WAC F21 }\end{array}$ & 2.73 & 320 & $\begin{array}{l}7-65 \\
7-65 \\
2-56\end{array}$ & 98 & $\begin{array}{l}2.03 \pm 0.06 \\
2.13 \pm 0.08 \\
1.92 \pm 0.07\end{array}$ \\
\hline $029 / 107$ & 09/04/2016 & $\begin{array}{l}\text { NAC F22 } \\
\text { NAC F24 } \\
\text { WAC F12 } \\
\text { WAC F21 }\end{array}$ & 2.97 & 17 & $\begin{array}{l}9-60 \\
9-60 \\
5-60 \\
5-60\end{array}$ & 89 & $\begin{array}{l}3.63 \pm 0.07 \\
3.63 \pm 0.07 \\
3.58 \pm 0.13 \\
3.58 \pm 0.18\end{array}$ \\
\hline
\end{tabular}




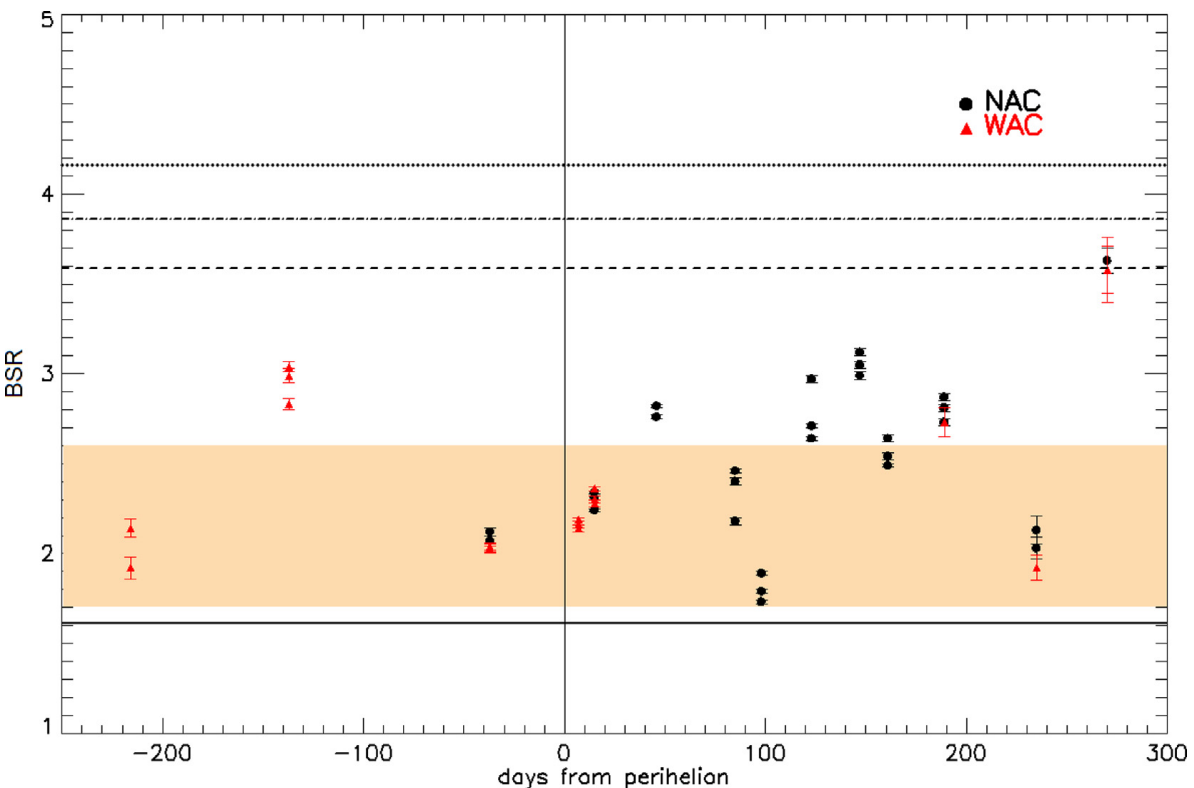

Figure 5. BSR versus time from perihelion. Colour band indicates the range of $B S R$ for cometary dust comae found in literature. Continuous, dashed, dot-dashed, and dotted lines correspond to interplanetary dust, 67P nucleus found in Hasselmann et al. (2017), Güttler et al. (2017), and Masoumzadeh et al. (2017), respectively.

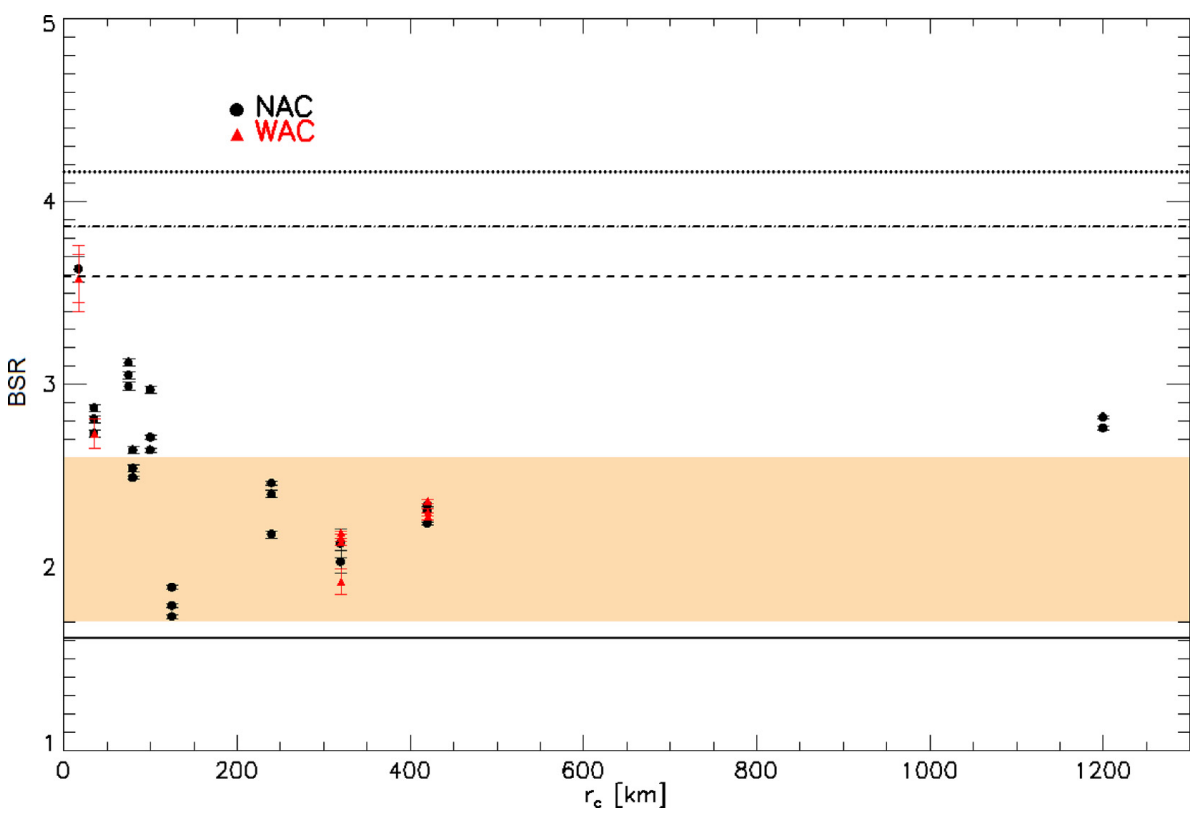

Figure 6. Post-perihelion $B S R$ versus nucleo-centric distance. Colour band indicates the range of $B S R$ for cometary dust comae found in literature. Continuous, dashed, dot-dashed, and dotted lines correspond to interplanetary dust, 67P nucleus found in Hasselmann et al. (2017), Güttler et al. (2017), and Masoumzadeh et al. (2017), respectively.

zero. According to Zakharov et al. (2018b), we have

$A_{\theta}=-\frac{3 Q_{\mathrm{g}}(1+e \cos \theta)}{4 \pi a\left(1-e^{2}\right) \rho_{\mathrm{C}} d_{\mathrm{C}}} \sqrt{\frac{\gamma+1}{\gamma-1}}$,

where $Q_{\mathrm{g}}$ is the gas loss rate from the nucleus, $d_{\mathrm{C}}$ and $\rho_{\mathrm{C}}$ are the chunk diameter and bulk density, and $\gamma$ is the specific heat ratio of the coma gas. Here $\gamma$ is the vibrationally relaxed value $(\gamma=4 / 3)$ for water vapour (Crovisier 1984; Crifo \& Rodionov 1997). The $a$ and $e$ decrease per orbit becomes

$$
\begin{aligned}
& \Delta a=-\frac{3 Q_{\mathrm{g}}}{\rho_{\mathrm{C}} d_{\mathrm{C}}} \sqrt{\frac{(\gamma+1)\left(1+e^{2} / 2\right)^{2} a}{(\gamma-1)\left(1-e^{2}\right)^{3} \mathrm{GM}}} \\
& \Delta e=-\frac{9 e Q_{\mathrm{g}}}{4 \rho_{\mathrm{C}} d_{\mathrm{C}}} \sqrt{\frac{\gamma+1}{(\gamma-1)\left(1-e^{2}\right) a \mathrm{GM}}} .
\end{aligned}
$$

With $Q_{\mathrm{g}} \approx 500 \mathrm{~kg} \mathrm{~s}^{-1}$, being this a mean perihelion value (Shinnaka et al. 2017), $\rho_{\mathrm{C}} \approx 500 \mathrm{~kg} \mathrm{~m}^{-3}$ (Preusker et al. 2017), and $d_{\mathrm{C}} \approx$ 
$0.2 \mathrm{~m}$ (Pajola et al. 2017), we get $\Delta a=-1.2 \mathrm{~km}$ per orbit if $e=0.7$ (pericentre of $15 \mathrm{~km}$ ), $\Delta a=-1.8 \mathrm{~km}$ per orbit if $e=0.8$ (pericentre of $10 \mathrm{~km}$ ), and $\Delta a=-5 \mathrm{~km}$ per orbit if $e=0.9$ (pericenter of $5 \mathrm{~km}$ ). The $e$ decrease can be neglected because $|\Delta e|<0.01$ per orbit if $e$ $<0.98$. The chunk orbit collapses on the nucleus in a few months if $0.8<e<0.98$, as it is always the case (Fulle 1997). If $e=0.9$, the chunk collides with the nucleus with an orbital period of about one week when $a=20 \mathrm{~km}$. With an average $Q_{\mathrm{g}}=100 \mathrm{~kg} \mathrm{~s}^{-1}$ between perihelion and equinox, $\Delta a$ becomes about $1 \mathrm{~km}$ per orbit and 30 orbits are about 10 months, not far from the time from perihelion to equinox (Hansen et al. 2016).

This explains why Rosetta observes BSR larger than the aforementioned threshold when it is inside the chunk cloud slowly collapsing on the nucleus after perihelion, where the light scattering is also coming from chunks. Since the orbital radial velocity at the nucleus distance $r$ is

$v_{r}=\sqrt{\frac{G M}{a r^{2}}\left[e^{2} a^{2}-(a-r)^{2}\right]}$

then the probability to observe an orbiting chunk at the distance $r$ is

$p(r, a, e)=\frac{r / a}{\pi \sqrt{e^{2}-(1-r / a)^{2}}}$.

Numerical integrations of equation (8) with $a$ and $e$ evolving according to equations (5) and (6) show that a cloud of orbiting chunks collapsing on to the nucleus has a space density depending approximately on $1 / r^{2}$, i.e. the same of an isotropic dust expanding flow. This occurs for a chunk supply vanishing from perihelion to the outbound equinox.

The presence of a cloud of dm-sized junks close to the nucleus can be corroborated with complex 3D models of the cometary coma and intensity scattering calculations using the complete ensemble of the dust size distribution. At present several authors of this manuscript are involved in using the recently published RZC coma model (Zakharov et al. 2018a) to follow this approach. The results of this investigation will be the subject of separate future papers.

\section{SUMMARY AND CONCLUSIONS}

Four OSIRIS image series were taken from 2015 January to 2016 April to investigate the backscattering behaviour of the $67 \mathrm{P}$ dust coma at low phase angles. We added to our analysis also the series described in Bertini et al. (2017) obtained starting from larger phase angles to increase the temporal resolution of our findings.

We measured a $B S R$, defined as the ratio of the reflectivity at $0^{\circ}$ and $30^{\circ}$ of phase angle, in the range $\sim[1.7-3.6]$, broader than the range found in literature from ground-based observations of other comets.

We found a clear trend considering the dust BSR and the Rosetta distance $r_{\mathrm{c}}$ from the nucleus centre during the post-perihelion phase, being the dust $B S R$ larger that the classical values for cometary dust only for nucleocentric distances smaller than $\sim 100 \mathrm{~km}$, with the largest $B S R$ value, close to the nucleus one, corresponding to the smallest nucleocentric distance $(17 \mathrm{~km})$.

We explain this trend in terms of a cloud of chunks orbiting the nucleus at distances $<100 \mathrm{~km}$ ejected during perihelion and slowly collapsing on the nucleus on times scales of few months because of the coma gas drag. The presence of large fragments close to the nucleus in order to explain similarities in the coma and nucleus phase functions was already suggested as a possibility in the paper on the backscattering phase function of 67P in Fink \& Doose (2018).
The systematic BSR increase observed after perihelion at nucleus distances lower than about $100 \mathrm{~km}$ confirms the dominant transfer of dm-sized chunks from the southern nucleus hemisphere to the northern one (Mottola et al. 2015; Fulle et al. 2016a; Keller et al. 2017; Pajola et al. 2017). Our observations suggest that such a transfer involves much more than the 20 per cent of the total chunk mass emitted at perihelion estimated by Keller et al. (2017), because the chunks in bound orbits dominate the average dust cross-section of the $67 \mathrm{P}$ coma within a radius of $100 \mathrm{~km}$. We find that the process explaining such a dominant transfer is the gas drag perturbing all chunk bound orbits, never taken into account in previous fallout models (Thomas et al. 2015; Lai et al. 2016; Keller et al. 2017). Such gas drag makes the chunk fallout much more probable than any chunks escape from the nucleus gravity field, collapsing all chunk bound orbits on the nucleus within a few months, as observed. The scattering properties of these chunks, of sizes $\geq 0.1 \mathrm{~m}$ (Pajola et al. 2017), match those of the nucleus surface. This implies that the threshold size where the dust phase function (Bertini et al. 2017) becomes the nucleus phase function (Güttler et al. 2017) is somewhere between $2.5 \mathrm{~mm}$ (Fulle et al. 2018) and $0.1 \mathrm{~m}$ (Pajola et al. 2017). It also implies that the chunk loss rates and size distributions estimated by Fulle et al. (2016b) and Ott et al. (2017) are not affected by any bias due to an improperly assumed phase function.

\section{ACKNOWLEDGEMENTS}

Optical, Spectroscopic, and Infrared Remote Imaging System (OSIRIS) was built by a consortium of the Max-Planck-Institut für Sonnensystemforschung, Göttingen, Germany, Centro di Ateneo di Studi e Attività Spaziali (CISAS) - University of Padova, Italy, the Laboratoire d' Astrophysique de Marseille, France, the Instituto de Astrofísica de Andalucia, Consejo Superior de Investigaciones Científicas (CSIC), Granada, Spain, the Research and Scientific Support Department of the European Space Agency, Noordwijk, The Netherlands, the Instituto Nacional de Técnica Aeroespacial, Madrid, Spain, the Universidad Politéchnica de Madrid, Spain, the Department of Physics and Astronomy of Uppsala University, Sweden, and the Institut für Datentechnik und Kommunikationsnetze der Technischen Universität Braunschweig, Germany. The support of the national funding agencies of Germany (DLR: Deutschen Zentrums für Luft-und Raumfahrt), France (CNES: Centre national d'études spatiales), Italy (ASI: Agenzia Spaziale Italiana), Spain (MEC: Ministry of Education \& Science), Sweden (SNSB: Swedish National Space Board), and the European Space Agency (ESA) Technical Directorate is gratefully acknowledged.

\section{REFERENCES}

Acton C., Bachman N., Semenov B., Wright E., 2018, Planet. Space Sci., 150,9

Acton C. H., 1996, Planet. Space Sci., 44, 65

Agarwal J. et al., 2016, MNRAS, 462, S78

Bertini I., Thomas N., Barbieri C., 2007, A\&A, 461, 351

Bertini I. et al., 2017, MNRAS, 469, S404

Crifo J. F., Rodionov A. V., 1997, Icarus, 127, 319

Crovisier J., 1984, A\&A, 130, 361

Della Corte V. et al., 2014, J. Astron. Instrum., 3, 1350011

Dohlen K., Jorda L., Lamy P., Toth I., Origne A., 2010, in Oschmann J. M., Jr, Clampin M. C., MacEwen H. A., eds, Proc. SPIE Conf. Ser. Vol. 7731, Space Telescopes and Instrumentation 2010: Optical, Infrared, and Millimeter Wave. SPIE, Bellingham, p. 773149

Fink U., Doose L., 2018, Icarus, 309, 265 
Fornasier S. et al., 2015, A\&A, 583, A30

Frattin E., et al., 2018, MNRAS, 1, 1

Fulle M., 1997, A\&A, 325, 1237

Fulle M., Altobelli N., Buratti B., Choukroun M., Fulchignoni M., Grün E., Taylor M. G. G. T., Weissman P., 2016a, MNRAS, 462, S2

Fulle M. et al., 2016b, ApJ, 821, 19

Fulle M. et al., 2018, MNRAS, 476, 2835

Gerig S.-B. et al., 2018, Icarus, 311, 1

Güttler C. et al., 2017, MNRAS, 469, S312

Hansen K. C. et al., 2016, MNRAS, 462, S491

Hasselmann P. H. et al., 2017, MNRAS, 469, S550

Ishiguro M., Yang H., Usui F., Pyo J., Ueno M., Ootsubo T., Minn Kwon S., Mukai T., 2013, ApJ, 767, 75

Keller H. U. et al., 2007, Space Sci. Rev., 128, 433

Keller H. U. et al., 2017, MNRAS, 469, S357

Kelley M. S., Lindler D. J., Bodewits D., A'Hearn M. F., Lisse C. M., Kolokolova L., Kissel J., Hermalyn B., 2013, Icarus, 222, 634

Lai I.-L. et al., 2016, MNRAS, 462, S533

Masoumzadeh N. et al., 2017, A\&A, 599, A11

Meech K. J., Jewitt D. C., 1987, A\&A, 187

Millis R. L., Ahearn M. F., Thompson D. T., 1982, AJ, 87, 1310

Moreno F., Pozuelos F., Aceituno F., Casanova V., Sota A., Castellano J., Reina E., 2012, ApJ, 752, 136

Moreno F. et al., 2018, AJ, 156, 237

Mottola S. et al., 2015, Science, 349, aab0232-1

Ott T. et al., 2017, MNRAS, 469, S276

Pajola M. et al., 2017, MNRAS, 469, S636

Preusker F. et al., 2017, A\&A, 607, L1

Rotundi A. et al., 2015, Science, 347, aaa3905

Shinnaka Y. et al., 2017, AJ, 153, 76

Thomas N. et al., 2015, A\&A, 583, A17

Tubiana C. et al., 2015, A\&A, 583, A46

Zakharov V. V., Crifo J.-F., Rodionov A. V., Rubin M., Altwegg K., 2018a, A\&A, 618, A71

Zakharov V. V., Ivanovski S. L., Crifo J.-F., Della Corte V., Rotundi A., Fulle M., 2018b, Icarus, 312, 121

${ }^{1}$ Department of Physics and Astronomy 'G. Galilei', University of Padova, Vicolo dell' Osservatorio 3, I-35122 Padova, Italy

${ }^{2}$ Astronomical Observatory of Trieste - INAF, Via Tiepolo 11, I-34143 Trieste, Italy

${ }^{3}$ Max Planck Institute for Solar System Research, Justus-von-Liebig-Weg 3, D-37077 Göttingen, Germany

${ }^{4}$ Institute of Astrophysics of Andalusia - CSIC, Glorieta de la Astronomia s/n, E-18008 Granada, Spain

${ }^{5}$ Deutsches Zentrum für Luft- und Raumfahrt (DLR), Institut für Planetenforschung, Rutherfordstrasse 2, D-12489 Berlin, Germany
${ }^{6}$ Istituto di Astrofisica e Planetologia Spaziali, INAF, Via fosso del cavaliere 100, I-00133 Rome, Italy

${ }^{7}$ Dipartimento di Scienze e Tecnologie, Universita degli Studi di Napoli Parthenope, CDN, IC4, I-80143 Naples, Italy

${ }^{8}$ Astronomical Observatory of Padova - INAF, Vicolo dell'Osservatorio 5 , I-35122 Padova, Italy

${ }^{9}$ Center of Studies and Activities for Space, CISAS, 'G. Colombo', University of Padova, Via Venezia 15, I-35131 Padova, Italy

${ }^{10}$ Physics Department, Auburn University, Auburn, AL 36849, USA

${ }^{11}$ MTA CSFK Konkoly Observatory, Konkoly Thege M. ut 15-17, H1121 Budapest, Hungary

${ }^{12}$ Aix Marseille Universite, CNRS LAM (Laboratoire d'Astrophysique de Marseille), UMR 7326, F-13388 Marseille, France

${ }^{13}$ Department of Physics and Astronomy 'G. Galilei', University of Padova, Via Marzolo 8, I-35131 Padova, Italy

${ }^{14}$ CNR-IFN UOS Padova LUXOR, Via Trasea 7, I-35131 Padova, Italy

${ }^{15}$ Laboratoire Atmosphères, Milieux et Observations Spatiales, CNRS \& Université de Versailles Saint-Quentin-en-Yvelines, 11 Boulevard d'Alembert, F-78280 Guyancourt, France

${ }^{16}$ Centro de Astrobiologia, CSIC-INTA, E-28850 Torrejon de Ardoz, Madrid, Spain

${ }^{17}$ International Space Science Institute, Hallerstrasse 6, CH-3012 Bern, Switzerland

${ }^{18}$ Scientific Support Office, European Space Research and Technology Centre/ESA, Keplerlaan 1, Postbus 299, NL-2201 AZ Noordwijk ZH, the Netherlands

${ }^{19}$ Jet Propulsion Laboratory, M/S 183-301, 4800 Oak Grove Drive, Pasadena, CA 91109, USA

${ }^{20}$ LESIA, Observatoire de Paris, Université PSL, CNRS, Univ. Paris Diderot, Sorbonne Paris Cité, Sorbonne Université, 5 Place J. Janssen, F-92195 Meudon Pricipal Cedex, France

${ }^{21}$ Department of Industrial Engineering, University of Padova, Via Venezia 1, I-35131 Padova, Italy

${ }^{22}$ Department of Industrial Engineering, University of Trento, Via Sommarive 9, I-38123 Trento, Italy

${ }^{23}$ Graduate Institute of Astronomy, National Central University, 300 ChungDa Rd, Chung-Li 32054, Taiwan

${ }^{24}$ Space Science Institute, Macau University of Science and Technology, Macao 999078, China

${ }^{25}$ Institut für Geophysik und extraterrestrische Physik (IGEP), Technische Universität Braunschweig, Mendelssohnstrasse 3, D-38106 Braunschweig, Germany

${ }^{26}$ Department of Geosciences, University of Padova, via G. Gradenigo 6, I-35131 Padova, Italy

This paper has been typeset from a $\mathrm{T}_{\mathrm{E}} \mathrm{X} / \mathrm{LT} \mathrm{E} \mathrm{X}$ file prepared by the author 